\title{
PEG10 is associated with treatment-induced neuroendocrine prostate cancer
}

\author{
Soojin Kim¹, Daksh Thaper1,2, Samir Bidnur², Paul Toren¹, Shusuke Akamatsu1, Jennifer L Bishop1, Colin Colins ${ }^{1,2}$, \\ Sepideh Vahid ${ }^{1}$ and Amina Zoubeidi1,2
}

'Vancouver Prostate Centre, Vancouver, British Columbia, Canada

2Department of Urologic Sciences, University of British Columbia, Vancouver, British Columbia, Canada

Correspondence should be addressed to A Zoubeidi: azoubeidi@prostatecentre.com

\begin{abstract}
Neuroendocrine (NE) differentiation of advanced prostate adenocarcinoma following androgen receptor (AR) axis-directed therapy is becoming increasingly recognized. Several models of this transdifferentiation provide insight into its molecular pathogenesis and have highlighted the placental gene PEG10 for further study. Using our unique model of enzalutamide resistance (ENZR) and NE differentiation, we studied PEG10/AR interplay in enzalutamide treatment-resistant cell lines 42DENZR and 42FENZR compared to LNCaP and castration-resistant 16DCRPC cells. ENZR cell lines with positive terminal NE marker status also displayed higher baseline expression of PEG10 compared to LNCaP and 16DCRPC. Antagonism of AR activity increased PEG10 expression followed by an increase in terminal NE markers. Conversely, stimulating AR activity via androgen supplementation reversed PEG10 and NE marker expression in a time and dose-dependent manner. These results were supported by human data showing that PEG10 expression is highest in NEPC and that AR-dependent gene, PSA, is negatively correlated with PEG10 in adenocarcinoma. Further, ChIP assay confirmed binding of activated AR to the PEG10 enhancer, decreasing PEG10 expression. While PEG10 did not drive NEPC, its knockdown reduced NE markers in our cell lines. Moreover, PEG10 knockdown in vitro- and in vivo-attenuated tumor growth. Overall, these observations indicate that PEG10 is an AR-repressed gene which modulates NE markers in ENZR cells and targeting PEG10 in advanced prostate cancer with NE features is a rational and viable option.
\end{abstract}

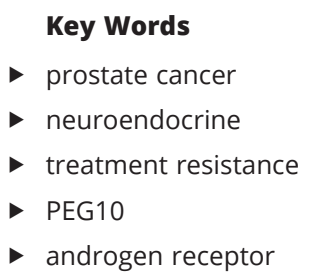

Journal of Molecular Endocrinology (2019) 63, 39-49

\section{Introduction}

Prostate cancer (PCa) is the most commonly diagnosed and the third leading cause of cancer mortality among Canadian men (CCSsACoC 2017). Initially, most cases of prostate adenocarcinoma are hormone driven and respond to androgen deprivation therapy (ADT); however, treatment resistance is inevitable and tumors progress to castration-resistant PCa (CRPC) (Davies et al. 2018). Persistent androgen receptor (AR) activity continues to be a driving force behind disease progression in CRPC and consequently, second-generation AR antagonists such as enzalutamide (ENZ) have demonstrated an overall survival benefit in CRPC, highlighting the ongoing 'targetable' nature of the AR even in advanced disease (Scher et al. 2012). Unfortunately, with the wider use of this agent, enzalutamide resistance (ENZR) is an increasingly common clinical entity. In some cases it may be mediated by AR mutations creating an agonist relationship between enzalutamide and the AR ligand-binding domain (LBD) 
(Balbas et al. 2013, Joseph et al. 2013, Korpal et al. 2013, Li et al. 2013). In other cases, after multi-agent AR-axis inhibition, aggressive CRPC cancers demonstrate a nonAR-driven phenotype with neuroendocrine (NE) features absent in typical PCa and CRPC (Wright et al. 2003, Akamatsu et al. 2015, Bishop et al. 2017). This has given rise to the term treatment-induced NE PCa (tNEPC) (Terry \& Beltran 2014, Aggarwal et al. 2018). The cellular origin of tNEPC is actively debated but increasing evidence suggests a 'transdifferentiation' from an AR-positive PCa to an AR-negative NE state characterized by minimal AR-dependent gene activity (e.g. PSA) and an increase in NE marker expression such as synaptophysin (SYP), chromogranin A (CHGA), neural cell adhesion molecule 1 (NCAM1) and neuron-specific enolase (NSE, gene symbol: ENO2) (Beltran et al. 2014, Bishop et al. 2017).

A number of molecular mechanisms were proposed to facilitate the progression of CRPC to NEPC (e.g. amplification of MYCN and AURKA; Beltran et al. 2011, loss of RB or PTEN; Tzelepi et al. 2012, Tan et al. 2014 and overexpression of epigenetic modulator EZH2; Dardenne et al. 2016). More importantly, the AR still plays a crucial, yet to be completely deciphered, role in NEPC (Bishop et al. 2015). Indeed, loss of AR activity and acquisition of a NE phenotype under the pressure of ENZ has raised the question if $\mathrm{AR}$ can act as a repressing agent on NE-like phenotype, a potential theory which is tested by mechanistically studying the interplay between AR- and NE-driving forces such as neural differentiation regulator BRN2 (Bishop et al. 2017) or growth-promoting paternally expressed gene 10 (PEG10) (Akamatsu et al. 2015).

Utilizing a unique in vivo-derived model of ENZ resistance (Kuruma et al. 2013, Bishop et al. 2017), our lab further explored the role of PEG10 in tNEPC as well as the interplay between PEG10 and AR in non-AR driven ENZR cells $\left(42 \mathrm{D}^{\mathrm{ENZR}}, 42 \mathrm{~F}^{\mathrm{ENZR}}\right)$ that display NE features compared to original $\mathrm{LNCaP}$ and $16 \mathrm{D}^{\mathrm{CRPC}}$ cells. Here, we elucidate the AR/PEG10 interplay by demonstrating that the AR binds to the PEG1O downstream enhancer and suppresses PEG10 expression. Moreover, we characterize the changes in PEG10 and NE marker expression in LNCaP, 16DCRPC, $42 \mathrm{D}^{\mathrm{ENZR}}$ and 42FENZR following androgen depletion and supplementation. We also silenced PEG10 both in vitro and in vivo to further delineate its association with $\mathrm{NE}$ marker expression and tumor growth, respectively. PEG10 silencing affects cell proliferation, confirming its progrowth properties (Akamatsu et al. 2015). Together, these findings endorse a mechanism whereby AR acts to repress PEG10 expression and also reveal further evidence for PEG10 involvement in NEPC progression. This study also highlights the utility of our novel ENZR cell model, which by mimicking the heterogeneity of ENZ resistance, permits evaluation of mechanistic features of NEPC progression and provides a future platform for studying NEPC drug targets, including PEG10.

\section{Materials and methods}

\section{Generation of ENZR xenografts and cell lines}

The detailed procedure for generation of CRPC and ENZR cell lines can be found in our previously published report (Bishop et al. 2017).

\section{Cell culture and transfection}

LNCaP cells were kindly provided by Dr Leland W.K. Chung (Emory University) and authenticated in January 2013 using short tandem repeat (STR) profile. LNCaP and castration-resistant LNCaP-derived V16D cell lines were grown in RPMI 1640 (Thermo Scientific) with 10\% FBS (Invitrogen), and enzalutamide-resistant $42 \mathrm{~F}$ and $42 \mathrm{D}$ cell lines were grown in RPMI 10\% FBS along with $10 \mu \mathrm{M}$ Enzalutamide (Selleck Chemicals). All cell lines were grown at $37^{\circ} \mathrm{C}$ in a humidified air atmosphere with $5 \%$ $\mathrm{CO}_{2}$ and were kept at passages less than 25. For induction of AR activity experiments, R1881 (0-5 nM) was added to the cell lines after culturing the cells in RPMI with $10 \%$ CSS (Invitrogen) for 6 days prior. For transient loss of function experiments, Silencer Select siRNA PEG10 (siPEG10) (\#s23005, Thermofisher) and custom-designed control siRNA(siCTRL) 5'-AUCAAACUGUCAGCGCUG-3' (Dharmacon) were transfected twice to improve silencing using Oligofectamine (Invitrogen) at the final concentration of $20 \mathrm{nM}$ according to the manufacturer's protocol. The duration for the first transfection was 16 hours, and the duration of the second transfection $4 \mathrm{~h}$. Stable PEG10-knockdown cells were generated by transfecting PEG10 shRNA (Lentiviral Particles sc-152158-V (Santa Cruz)) and control shRNA (Lentiviral Particles-A sc-108080) into cell lines according to the manufacturer's protocol). After transfection, the cells were isolated via selection with $2.3 \mu \mathrm{g} / \mathrm{mL}$ puromycin (Gibco) containing media. Stably PEG10-knockdown cells were challenged with enzalutamide for 6 days prior to experiments to ensure knockdown of PEG10. PEG10 overexpression experiments were carried in LNCaP cells for 7 days using $1 \mu \mathrm{g}$ of full-length PEG10 plasmid (kindly provided by Dr Akamatsu) and TransIT®-2020 (Mirus Bio.). 


\section{Quantitative RT-PCR}

Total RNA was extracted using TRIzol (Invitrogen) and $2 \mu \mathrm{g}$ was reversed transcribed using MMLV reverse transcriptase and random hexamers (Invitrogen). Levels of PEG10, AR, PSA, NCAM1, NSE, SYP, CHGA and $G A P D H$ transcripts were quantified using FastStart Universal SYBR Green Master (Roche) with custom primers (listed in Supplementary Table 2, see section on supplementary data given at the end of this article). The average change in threshold cycle $(\Delta \mathrm{CT})$ relative to endogenous GAPDH levels was determined for each sample and compared to control. All experiments were performed in triplicate and mean standard error was determined. Student $T$ tests were performed to test for differences between treatments.

\section{Western blot analysis}

Total protein was extracted using RIPA lysis buffer and the concentration was determined by using Pierce BCA Protein Assay Kit (Thermo Scientific). Forty microgram protein were separated by SDS-PAGE and blotted to a nitrocellulose membrane. The membranes were probed with primary antibodies against PEG10 (Novus Biologicals: rabbit NBP2-13749 or mouse NBP1-28875) at 1:1000 and 1:500 dilution respectively, AR N-20 sc-816 (Santa Cruz) at 1:5000 dilution, and PSA C-19 sc-7638 (Santa Cruz) at 1:500 dilution. All antibodies were dissolved in 2.5\% bovine serum albumin + TBS-T solution and the membranes were incubated overnight at $4^{\circ} \mathrm{C}$. Vinculin MA5-1690 (Thermo Scientific) at 1:10,000 was blotted as a loading control. Proteins were visualized using the Odyssey system (Li-Cor Biosciences). Alternately, HRP detected the bands with Super Signal West Femto Maximum Sensitivity Substrate (Thermo Scientific).

\section{Proliferation assay}

Three thousand cells from each condition of the cell lines were plated onto 96-well plates in RPMI $+10 \% \mathrm{FBS}+2.3 \mu \mathrm{g} /$ $\mathrm{mL}$ puromycin, where ENZ-resistant cell line media was supplemented with enzalutamide $10 \mu \mathrm{M}$. Over the span of 8 days, the cells were fixed with 1\% glutaraldehyde and stained with crystal violet. Sorenson's buffer was used to solubilize the stain for absorbance measurement at $540 \mathrm{~nm}$ on the Epoch reader (BioTek). Mean optical density values of PEG10 shRNA cells were compared to those of control to derive relative cell proliferation percentages.

\section{AR chromatin immunoprecipitation (ChIP)}

All cell lines were cultured in RPMI $+10 \%$ CSS on $15 \mathrm{~cm}$ plates with or without $1 \mathrm{nM}$ R1881 for 4 days. The sequence for the AR-binding site is summarized in Supplementary Table 3 . The ChIP assay was performed using EZChIP kit (EMD Millipore) according to the manufacturer's protocol. Briefly, once the cells grew to $90 \%$ confluency, they were cross-linked with $1 \%$ formaldehyde and the cross-linking was quenched with $0.125 \mathrm{M}$ glycine. Upon cell collection, the nuclei were extracted and sonicated into $200 \mathrm{bp}$ fragments. The DNA-protein complex was immunoprecipitated with either $5 \mu \mathrm{g}$ AR N-20 sc-816 (Santa Cruz) or rabbit IgG overnight in $4^{\circ} \mathrm{C}$. The complex was washed with lowsalt buffer (0.1\% SDS, 1\% Triton X, 2 mM EDTA, $20 \mathrm{mM}$ Tris-HCl, $150 \mathrm{mM} \mathrm{NaCl})$, high-salt buffer (0.1\% SDS, $1 \%$ Triton $\mathrm{X}, 2 \mathrm{mM}$ EDTA, $20 \mathrm{mM}$ Tris- $\mathrm{HCl}, 500 \mathrm{mM}$ $\mathrm{NaCl}$ ), LiCl buffer (250 mM LiCl, 1\% NP40, 1\% sodium deoxycolate, $1 \mathrm{mM}$ EDTA, $10 \mathrm{mM}$ Tris- $\mathrm{HCl}$ ) and finally with TE buffer. The complex was reverse cross-linked and purified. The extracted DNA fragments were subjected to qRT-PCR using PEG10 primers that were designed to cover the predicted AR-binding site on the PEG10 enhancer region CACTTGCTGTTCTCGCCCTA (forward), AGGGGAAGCAAAGATGAGCC (reverse). The KLK3 enhancer primers (5'-TCTGCCTTTGTCCCCTAGAT-3' (forward), 5'-AACCTTCATTCCCCAGGACT-3' (reverse)) were used as a positive control for AR activity.

\section{In vivo tumor formation}

All experimental protocols used in this study were approved by the Canadian Council on Animal Care as well as the Animal Care Committee of University of British Columbia (certificate number A016-0246) and were carried out in accordance with institutional guidelines. Two million 42F cells stably transfected with either control or PEG10 shRNA were injected subcutaneously into the flank region of 4- to 6-week-old male athymic nude mice post castration. Mice were purchased from Jackson Labs and were kept in the animal facility of Jack Bell Research Centre where veterinarians and staff monitored the mice multiple times a week. Each experimental group consisted of ten mice. Tumor volume was measured once a week (length $\times$ width $\times$ depth $\times 0.5432$ ) until the tumor volume was $\geq 10 \%$ of body weight. Tumors were harvested for evaluation of gene expression and protein expression by qRT-PCR and Western blot respectively. 


\section{Statistical analysis and data representation}

Unpaired, two-tailed, Student's $T$ tests were performed to analyze statistical significance between groups using Graph Pad Prism (Graph Pad Software). Significance is indicated as follows: ${ }^{*} P<0.05,{ }^{*} P<0.01,{ }^{* *} P<0.001$, ${ }^{* * * *} P<0.0001$. Unless otherwise indicated, graphs show representative data from one of at least three independent experiments \pm S.E.M.

\section{Results}

\section{PEG10 is highly expressed in NE-like ENZ resistance and in human NEPC}

To investigate the clinical relevance of PEG10 in human NEPC, we assessed the level in prostatic hormone-naïve adenocarcinoma, CRPC and NEPC patients in a recently published cohort (Beltran et al. 2016) and found that PEG10 level was significantly increased in NEPC compared with CRPC or adenocarcinoma (Fig. 1A). PEG10 mRNA expression was also tested across our established ENZR cell lines as well as the NEPC tumor-derived NCIH660 cell line. Reflecting the clinical data, compared to adenocarcinoma LNCaP and castrate-resistant V16D ${ }^{\text {CRPC }}$ cells, PEG10 expression was highest in human NEPC cell line, NCIH660 cells, followed by cell lines with neuroendocrine-like features, 42D ENZR and 42FENZR (Bishop et al. 2017) (Fig. 1B). Interestingly, the expression levels of NE markers (ENO2, NCAM1, CHGA and SYP) correlate with PEG10 expression in these cell lines (Fig. 1C). Similar data were observed in human specimens from The Cancer Genome Atlas (TCGA) prostate cancer database (publicly available on https:// genome-cancer.ucsc.edu/) where $\mathrm{NE}$ representative markers ENO2, NCAM1, CHGA and CHGB were elevated in tumor samples with higher PEG10 expression (shown as red on heatmap) (Fig. 1D, left). Interestingly, analysis of the same samples showed that AR canonical targets PSA, FKBP5 and NKX3-1 are lower in tumors with high PEG10 expression (Fig. 1D, right). These findings were supported by more public data mining for the relationship between PEG10 and serum PSA from CRPC samples generated by Grasso et al. (2012) which further confirmed PSA/PEG10 inverse correlation suggesting that AR activity may be involved in PEG10 regulation (Fig. 1E). Furthermore, data from two other cohorts (Rajan et al. 2014, Beltran et al. 2017) showed that PEG10 is increased upon ADT (Supplementary Table 1).

\section{AR activity negatively regulates PEG10 in enzalutamide-resistant cell lines}

Following the observation from our ENZR model and inverse correlation between PEG10 and PSA from Grasso analysis (Fig. 1E), we tested the hypothesis if $\mathrm{AR}$ is a negative regulator of PEG10. We confirmed that in LNCaP cells, AR inhibition using $10 \mu \mathrm{M}$ ENZ leads to decreased PSA expression, while PEG10 expression is increased in a time-dependent manner at both the mRNA and protein levels (Fig. 2A). This is in harmony with previous data showing PEG10 is repressed by AR in LNCaP cells (Akamatsu et al. 2015). The effect of ENZ is accentuated in charcoal-stripped serum (CSS) media, with highest PEG10 expression occurring after 7 days of exposure to CSS + ENZ, which represents maximal androgen blockade condition. Similar results were reproduced in CRPC cells $16 \mathrm{D}^{\mathrm{CRPC}}$ (Supplementary Fig. 1A). It is important to note that PEG10 has several isoforms due to alternative translation initiation sites, alternative splicing, posttranslational
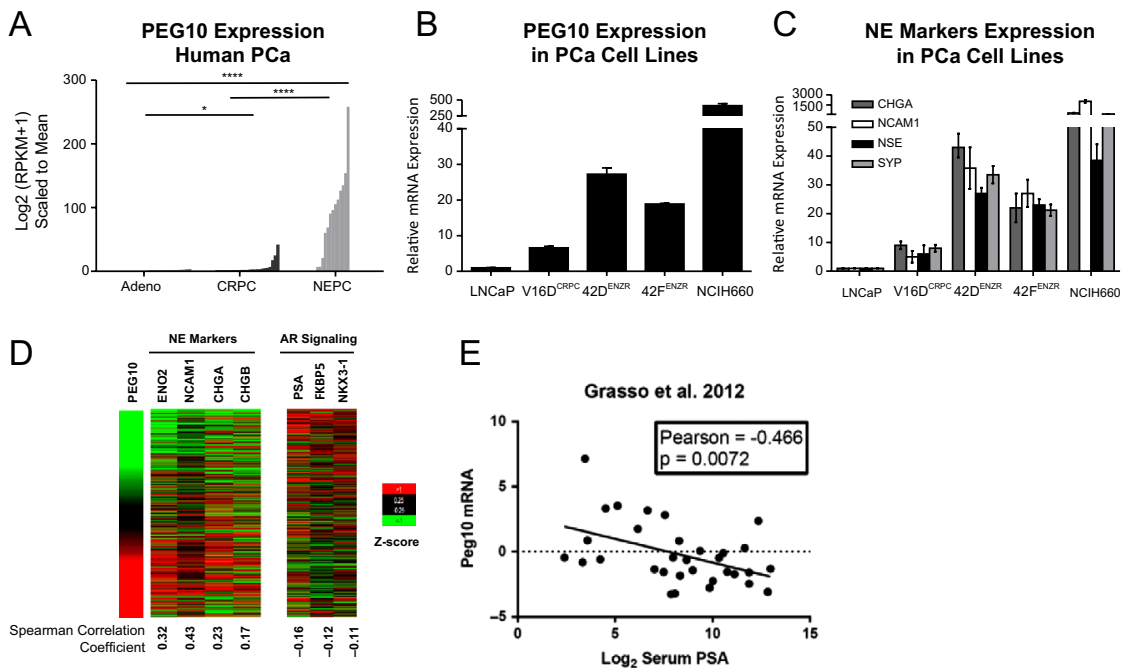

E

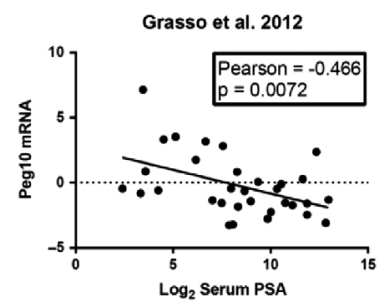

\section{Figure 1}

PEG10 is highly expressed in non-AR driven human NEPC, and ENZR cells feature increased levels of PEG10 and a NE differentiation signature. (A) PEG10 expression in Adeno $(n=37)$, CRPC $(n=34)$ and NEPC $(n=15)$ patients obtained from Beltran cohort (Beltran et al. 2016). (B) Relative mRNA expression of PEG10 and (C) relative mRNA expression of NE markers in V16DCRPC, 42DENZR, 42FENZR and NClH660 cells compared to LNCaP (=1). (D) Heat map of PEG10 and neuroendocrineassociated genes as well as canonical AR targets PSA, FKBP5 and NKX3-1 in RNA-sequencing data obtained from The Cancer Genome Atlas (TCGA) prostate cancer samples $(n=499)$. (E) PEG10 expression in CRPC tumors versus patient serum PSA (Grasso et al. 2012). Statistical significance is shown as $* P<0.05 ; * \star * * P<0.0001$. 
A
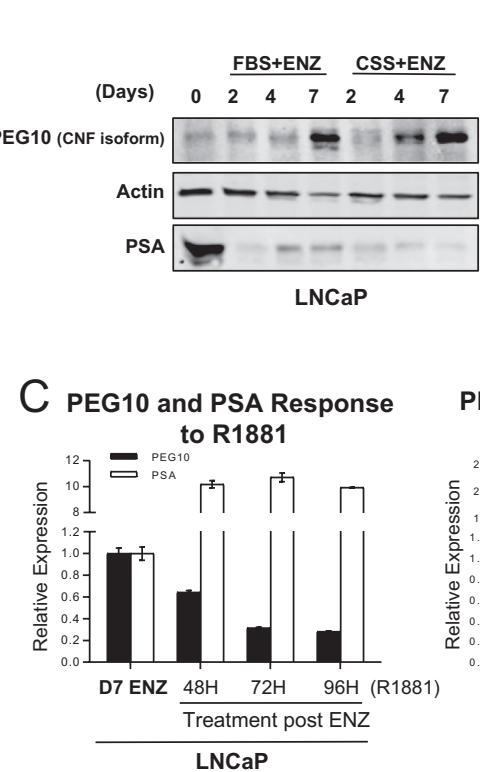

D NE markers response to

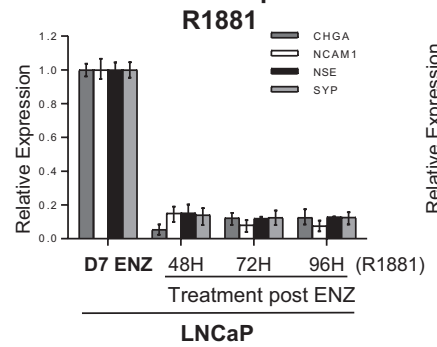

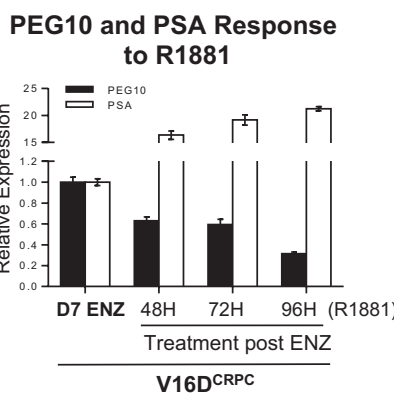

NE markers response to

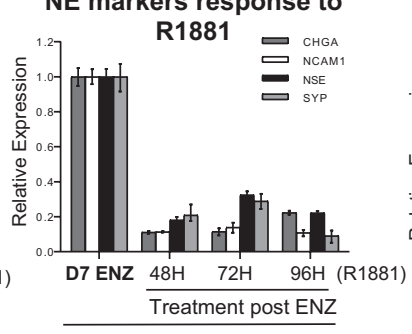

V16 ${ }^{\text {CRPC }}$
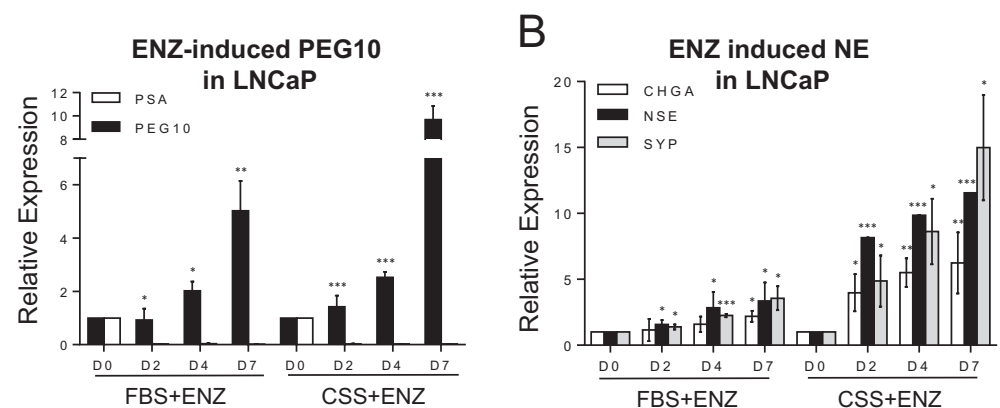

PEG10 and PSA Response to R1881

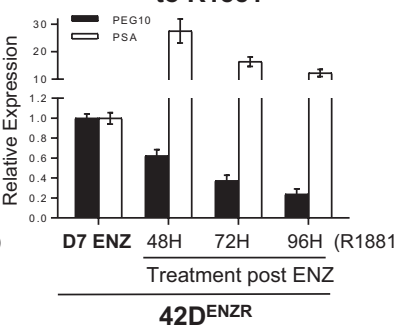

NE markers response to

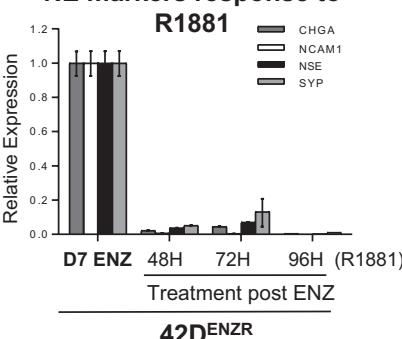

PEG10 and PSA Response to R1881

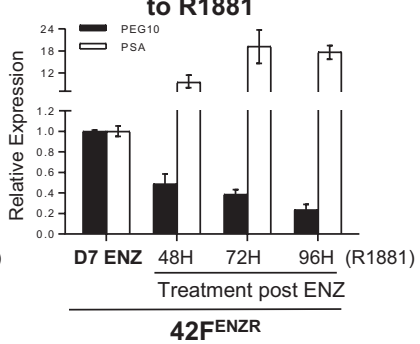

NE markers response to

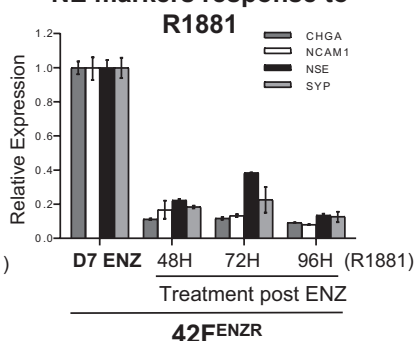

E 42D ENZR $-A R$ ChIP

42FENZR $-A R$ ChIP
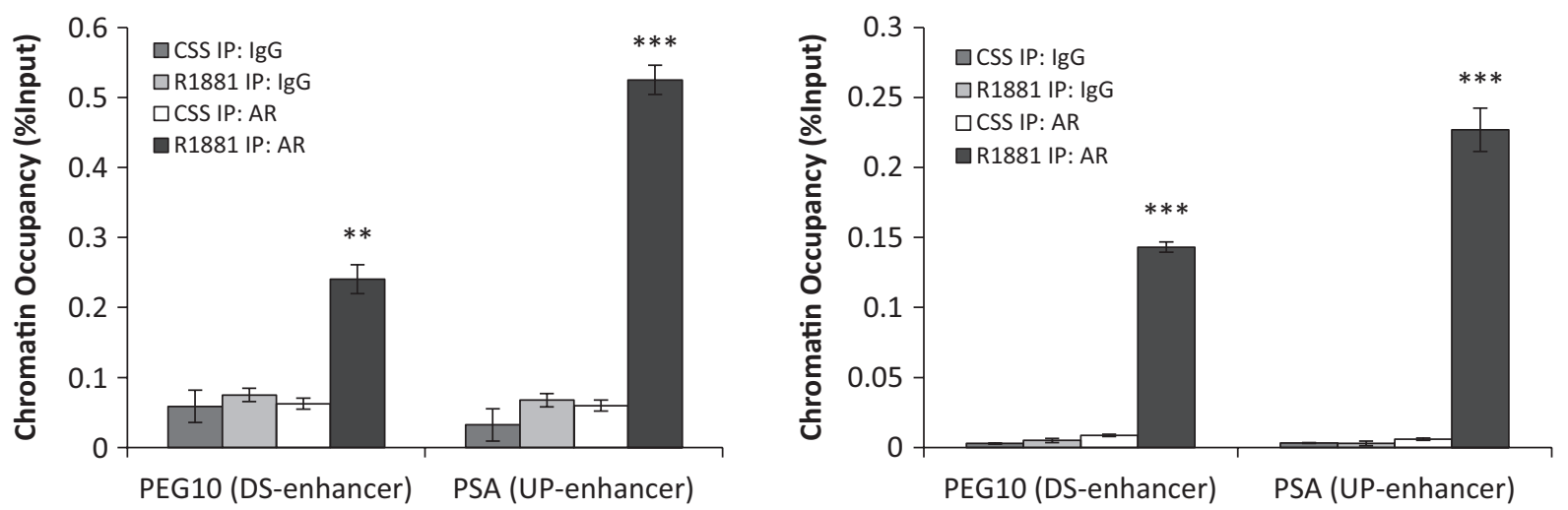

Figure 2

PEG10 and PEG10-dependent neuroendocrine marker expression are induced by ENZ and suppressed by reintroduction of AR activity. (A) Protein and relative mRNA expression of PEG10 and PSA in LNCaP cells treated $\pm 10 \mu \mathrm{mol} / \mathrm{L}$ ENZ for $2,4,7$ days compared with day 0 (=1) (Relative PSA expression after treatment is $<0.2$ ). (B) Relative mRNA expression of NE markers expression in LNCaP cells treated $10 \mu \mathrm{mol} / \mathrm{L}$ ENZ for 2, 4, 7 days compared with day $0(=1)$ in FBS or CSS. (C and D) Relative mRNA expression of PEG10, PSA and NE markers expression in LNCaP, V16DCRPC, 42DENZR and 42FENZR with reintroduction of androgen after 7 days of $10 \mu \mathrm{mol} / \mathrm{L}$ ENZ treatment $(=1)$. Graphs show representative data from three independent experiments. (E) Chromatin immunoprecipitation showing AR binding to the enhancer region of PEG10 in 42DENZR and 42FENZR treated with $10 \mathrm{nmol} / \mathrm{L}$ R1881 for $6 \mathrm{~h}$ non-treated control (=1); Corresponding PSA binding used as positive control. Graphs show pooled data from three independent experiments. 
proteolytic cleavage and -1 ribosomal frameshift translation. We were interested in looking at CNF band as it was previously shown to augment upon ENZ treatment (Akamatsu et al. 2015). Parallel to PEG10 expression, NE markers were increased in both FBS + ENZ and CSS +ENZ (Fig. 2B).

Inversely, we assessed the effects of AR stimulation with synthetic androgen (R1881) on PEG10 expression after 7 days of androgen deprivation. R1881 significantly reduced PEG10 mRNA levels across multiple cell lines (Fig. 2C) and was accompanied by a reduction in terminal NE markers (Fig. 2D). Interestingly, the PSA expression was recovered in all cell lines including 42D ENZR and 42FENZR in a time- and dose-dependent fashion (Fig. 2C and Supplementary Fig. 1B). Importantly, publicly available ChIP-seq data for AR shows a strong AR-binding peak in the enhancer region of the PEG10 gene (www. cistrome.org) (Supplementary Fig. 2). Indeed, using chromatin immunoprecipitation (ChIP) on our cell lines, 42DENZR and 42FENZR, we observed drastic increase in AR occupancy at this ARE after R1881 stimulation (Fig. 2E). PSA enhancer served as an AR-dependent positive control (Fig. 2E). Together, these results indicate that PEG10 expression is negatively regulated by AR activity in CRPC and ENZR cells. Also, the expression of NE markers is positively correlated to PEG10 in these cells.

\section{PEG10 is required for NE marker expression across multiple cell lines}

Using gain- and loss-of-function approaches, we investigated whether PEG10 is a driver of NEPC. We observed no significant upregulation of NE markers following PEG10 overexpression indicating that PEG10 is not a driver of NEPC (Fig. 3A and Supplementary Fig. 3). However, our previous data suggested that PEG10 is supporting a NE phenotype across cell lines. To confirm this, we generated transient and stable PEG10-knockdown conditions via small-interference (siRNA) and shorthairpin RNA (shRNA) respectively (Supplementary Fig. 4). Transient PEG10 knockdown (siPEG10) lead to a marked reduction in mRNA levels of CHGA, NCAM1, NSE and SYP in ENZ-treated LNCaP and V16DCRPC and also in 42D ${ }^{\text {ENZR }}$ and 42FENZR (Fig. 3B). Similar results were observed when shPEG10 prevented the ENZ-induced upregulation of NE markers (only the most effective clones are demonstrated) (Fig. 3C). Overall, these results confirm that although PEG10 is not a driver of NEPC, it is indeed important for maintaining the NE marker expression.

\section{PEG10 plays a role in cell proliferation in vitro and in vivo}

In addition to the expression of terminal NE markers, we questioned whether PEG10 is involved in regulating proliferation of cells. Previous studies have shown that PEG10 plays a role in breast and pancreatic cancer cell proliferation (Li et al. 2016, Peng et al. 2017). More importantly, Akamatsu et al. showed a supporting role for PEG10 in LNCaP and PC3 prostate cancer cells (Akamatsu et al. 2015) but not in NE-like prostate cells. Therefore, we investigated the effects of PEG10 knockdown on cell proliferation ENZ-treated LNCaP and V16DCRPC as well as $42 \mathrm{D}^{\mathrm{ENZR}}$ and $42 \mathrm{~F}^{\mathrm{ENZR}}$ cell lines. PEG10 knockdown significantly reduced proliferation across all cell lines in vitro (Fig. 4A). Similar data were observed in vivo where shPEG10 42FENZR murine xenograft was established with significant attenuation of tumor growth compared to control (Fig. 5A, left). Waterfall plot of tumor sizes between groups highlights four mice from the shPEG10 group which failed to grow any tumor beyond 10 weeks (Fig. 5A, right). Tumor tissue analysis confirms PEG10 knockdown and significantly reduced expression of CHGA, a representative NE marker (Figurer 5B). These in vitro and in vivo results suggest that PEG10 may be an ideal target to reduce tumor proliferation in ENZR.

\section{Discussion}

Although de novo NEPC is rare and includes less than 1\% of all diagnosed PCa cases, there is strong clinical evidence for the emergence of a more aggressive, treatmentinduced neuroendocrine phenotype after treatment with strong APIs such as enzalutamide (17\%) (Beltran et al. 2016, Aggarwal et al. 2018). While the mechanisms of such neuroendocrine transdifferentiation remain unclear, numerous putative targets have been identified as related to this process, including AURKA, MYCN, REST, BRN2 and PEG10 (Beltran et al. 2011, Akamatsu et al. 2015, Zhang et al. 2015, Bishop et al. 2017). How each of these proteins confers a change from an AR-positive adenocarcinoma to an AR-negative neuroendocrine phenotype, is only beginning to be elucidated. Our study builds up on previous findings that PEG10 is an AR-repressed gene and a potential therapeutic target in NEPC. Mining human data, we demonstrate that in NEPC tumors expression of NE markers are positively and expression of PSA is negatively correlated to PEG10. More importantly, utilizing an in vivo-derived model of ENZ resistance, we show for the first time that AR activity negatively regulates PEG10 
A

Overexpression of PEG10 in LNCaP

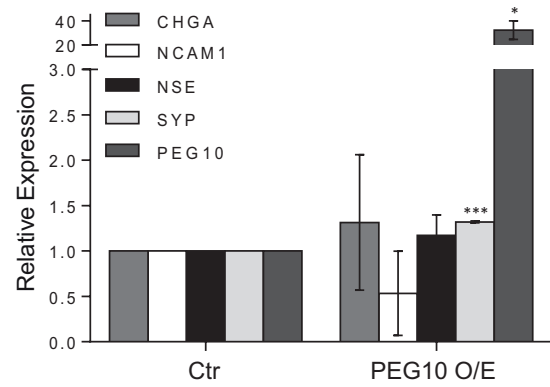

C
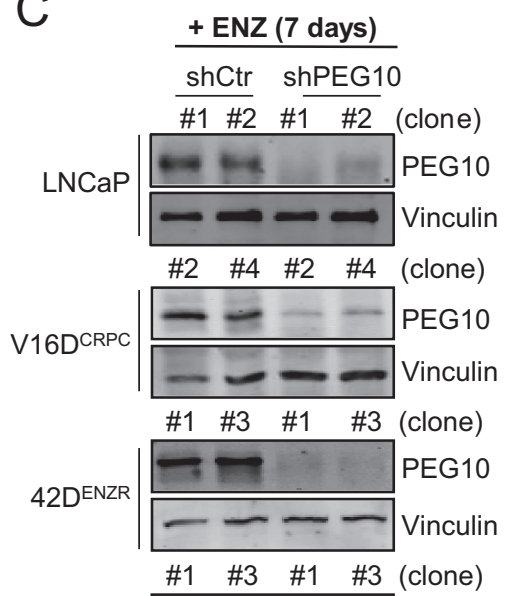

42FENZR \begin{tabular}{|l|l}
\hline & PEG10 \\
\hline$\ldots \ldots$ Vinculin
\end{tabular}
B PEG10 and NE markers: siPEG10 in LNCaP

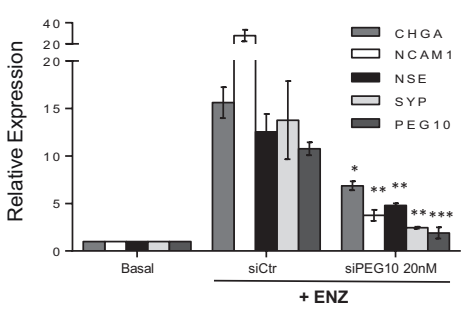

PEG10 and NE markers: siPEG10 in 42DENZR

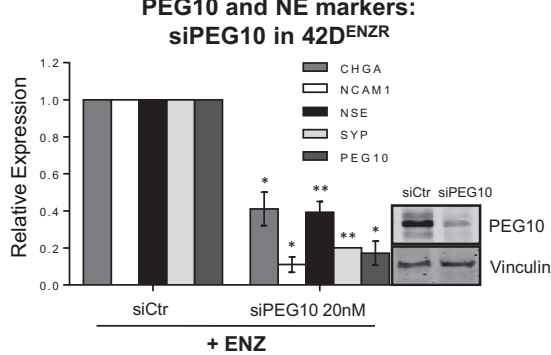

PEG10 and NE markers: shPEG10 in LNCaP

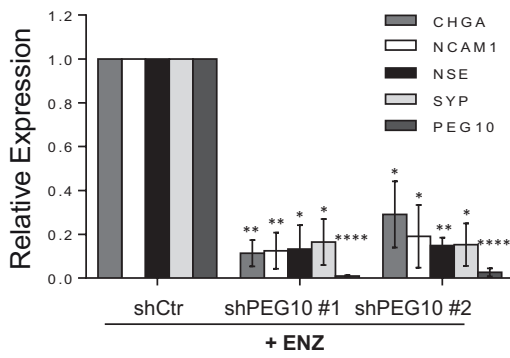

PEG10 and NE markers: ShPEG10 in 42DENZR

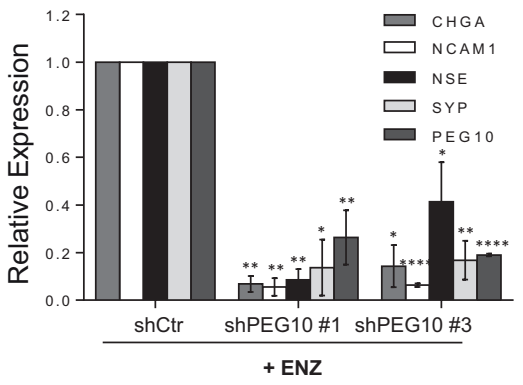

PEG10 and NE markers: siPEG10 in V16D CRPC

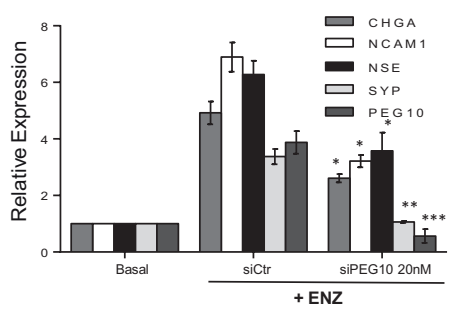

PEG10 and NE markers: siPEG10 in 42FENZR

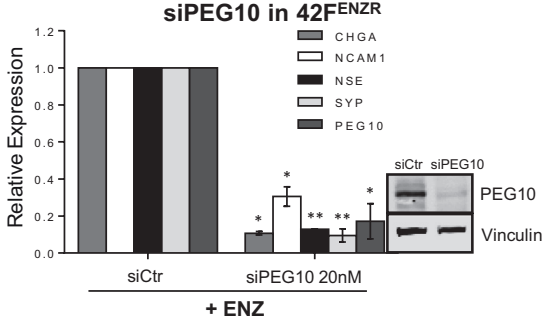

PEG10 and NE markers: shPEG10 in V16D CRPC

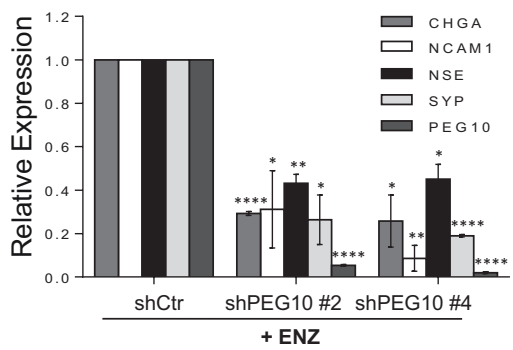

PEG10 and NE markers: shPEG10 in42FENZR

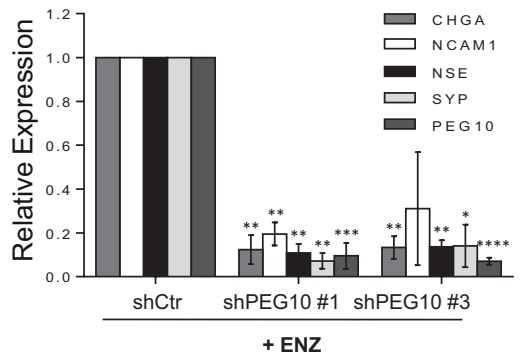

Figure 3

PEG10 is required to maintain NE marker expression across multiple cell lines. (A) Relative mRNA expression of PEG10 in LNCaP after overexpression compared with control (Ctr = 1). (B) (Top) Relative mRNA expression of PEG10 and NE markers in LNCaP and V16DCRPC transfected with PEG10 siRNA (siPEG10) or scrambled siRNA (siCtr) and treated with $10 \mu \mathrm{mol} / \mathrm{L}$ ENZ for $48 \mathrm{~h}$ compared to basal levels (basal = 1). Statistics show the decrease from siPEG10 to siCtr, (Bottom) Relative mRNA expression of PEG10 and NE markers and protein expression of PEG10 in 42DENZR and 42FENZR transfected with PEG10 siRNA (siPEG10) or scrambled siRNA. Western blots validate protein levels of PEG10 after knockdown. (C) (Left panel) PEG10 protein expression in stable PEG10 knockdown (shPEG10) compared with control-transfected cells (shCtr) in LNCaP, V16DCRPC, 42DENZR and 42FENZR cells maintained in $10 \mu \mathrm{mol} / \mathrm{L}$ ENZ for 7 days; (Right panel) Corresponding relative mRNA expression of PEG10 and NE markers compared with control $(\mathrm{shCtr}=1)$. Statistical analyses were performed on pooled data from at least three independent experiments. $* P<0.05 ; * \star P<0.01 ; * \star \star P<0.001$, $\star \star * \star P<0.0001$. 

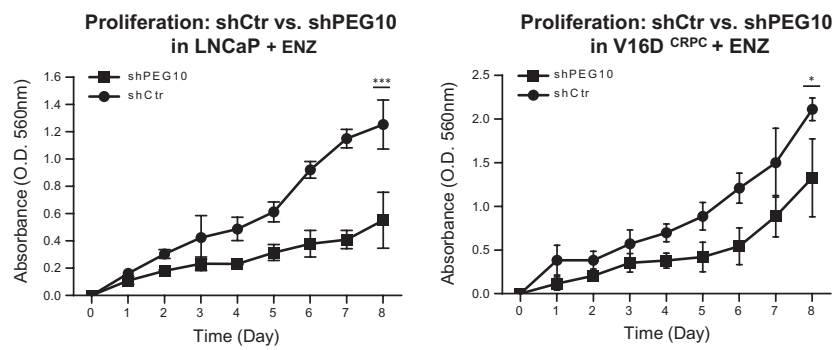

Proliferation: shCtr vs. shPEG10 in 42DENZR + ENZ
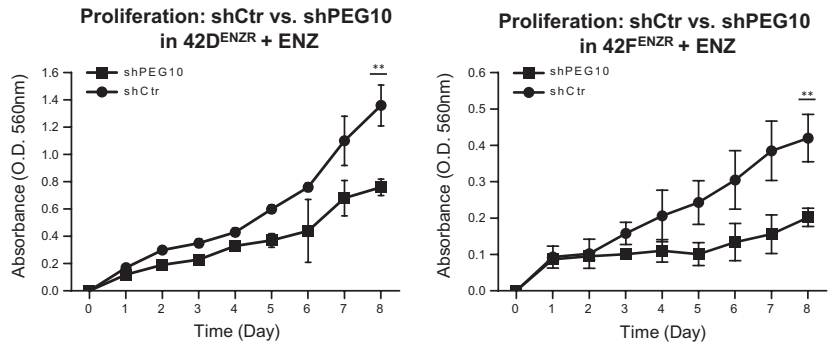

Figure 4

PEG10 promotes the proliferation of ENZR cell lines in vitro. Proliferation rate of shCtr and shPEG10 in LNCaP, V16DCRPC, 42DENZR and 42FENZR cells maintained in $10 \mu \mathrm{mol} / \mathrm{L}$ ENZ over the course of 8 days. Statistics were performed on pooled data from at least three independent experiments. $\star P<0.05 ; * \star P<0.01 ; * \star \star * P<0.001$.

expression in the context of ENZ resistance $\left(42 \mathrm{D}^{\mathrm{ENZR}}\right.$ and 42FENZR cells) as well as ENZ-treated adenocarcinoma cells (LNCaP and 16DCRPC). Finally, we show that PEG10 is required for maintaining NE phenotype in ENZR cells and targeting PEG10 reduces proliferation rate and expression of NE markers both in vitro and in vivo.
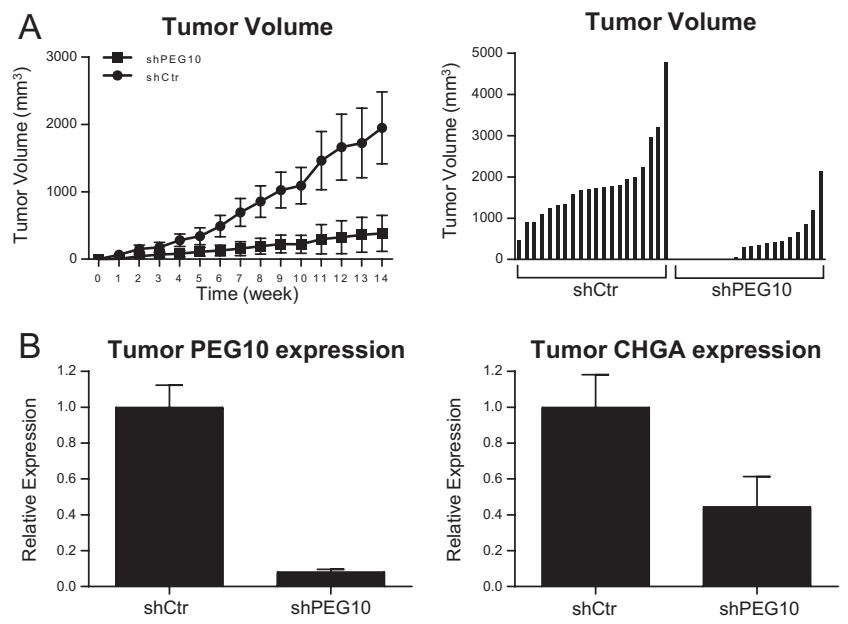

\section{Figure 5}

PEG10 knockdown via shPEG10 attenuates tumor growth in vivo. (A) (Left) Tumor volume of 42FENZR shCtr and shPEG10 xenografts grown in vivo ( $n=10)$, with (Right) representation in waterfall plot. Graph represents pooled data from five shPEG10 and five shCtr tumors. (B) Relative mRNA expression of PEG10 and representative NE marker chromogranin A (CHGA) in 42FENZR shPEG10 and shCtr xenografts $(=1)$, harvested at 12 weeks after inoculation. Values are expressed as mean \pm S.E.M.
PEG10 is an evolutionary conserved retrotransposonderived gene located within an imprinted domain on chromosome 7q21 and is highly expressed during embryogenesis while mostly silenced in adult tissues. It is required for placental development and murine PEG10 knockout results in early embryonic lethality due to placental defects (Ono et al. 2006). There is an increasing interest in genes with restricted expression during placental development as this phenomenon is observed in natural models of invasion as well as oncologic states (Rousseaux et al. 2013). During embryogenesis, such genes may serve pivotal roles in driving coordinated proliferation and invasion to allow proper embryonal development, while in adult cancers, reactivation of these genes especially in the context of inappropriate coordination may drive advanced disease phenotypes. PEG10 is suggested to be an inhibitor of apoptosis and mediator of proliferation and was shown to be overexpressed in several malignancies including acute and chronic lymphocytic leukemia, hepatocarcinoma, pancreatic cancer (Ribarska et al. 2012) and small cell bladder cancer (Robertson et al. 2017). In prostate cancer, our collaborators identified PEG10 as one of the upregulated genes in a human xenograft model that recapitulated transdifferentiation of adenocarcinoma to NEPC. Analysis of two independent cohorts revealed that PEG10 expression is elevated upon castration, and further significantly upregulated in clinical NEPC. In vitro mechanistic experiments demonstrated PEG10's role in promoting a proliferative, invasive phenotype as a result of direct transcriptional upregulation by E2F1 (major transcription factor in the RB signaling pathway) in the context of TP53 loss and enhanced TGF-beta signaling (Akamatsu et al. 2015). This is in harmony with results of a recent study which showed PEG10 is regulated by E2F1 and its overexpression promotes cell proliferation, migration and invasion in pancreatic cancer (Peng et al. 2017). Akamatsu et al. also reported PEG10 promoter occupancy by AR in LNCaP cells. However, the relation between PEG10 and AR was not delineated further as the majority of their experiments were carried out in PC3 and DU145 cells.

Despite the fact that tNEPC exhibits an AR-independent characteristic with low PSA production, there is little doubt that AR still plays a significant role in the emergence of NEPC. PC3 and DU145 cells have mutant TP53, express higher endogenous PEG10 compared to LNCaP and provide a good model for studying TGF-beta signaling; however, they are unable to accurately mimic NEPC as they do not express AR. AR is a regulator of cell plasticity and was suggested to be a 
repressor of several NEPC-promoting genes (Bishop et al. 2015, Akamatsu et al. 2018). Therefore, to understand the true role of PEG10 in INEPC, it is imperative to study it in an AR-positive model under the pressure of an AR inhibitor. Indeed, we confirmed that AR not only binds to the enhancer region of PEG10 in LNCaP, V16DCRPC, $42 \mathrm{D}^{\mathrm{ENZR}}$ and $42 \mathrm{~F}^{\mathrm{ENZR}}$ cells but that treatment with synthetic androgen R1881 increases AR occupancy. We also searched TCGA human data to provide clinical evidence for the role of PEG10 in NEPC. More importantly, analysis of prostate adenocarcinoma data available from Grasso et al. demonstrated that PGE10 expression is negatively correlated to PSA, AR's classic target gene. Also, a recent study on metastatic CRPC showed PEG10 is upregulated in cases treated with neoadjuvant docetaxel and androgen deprivation before going through radical prostatectomy (Beltran et al. 2017). Using systematic experiments, we elucidate this interaction in more detail by showing androgen deprivation increases PEG10 expression, while AR stimulation decreases PEG10. These findings highlight the adaptive nature of the AR axis present in this disease state and once again demonstrate how maximum AR inhibition not only reduces AR transcription activity but also removes AR-dependent transcriptional repression and consequently enhances other signaling pathways including NE differentiation.

Insight into tNEPC, specifically its development from a conventional adenocarcinoma, is an increasingly recognized clinical scenario that needs further investigation. Early studies were pivotal in identifying that the expression of NE markers in PCa was inversely related to AR activity (Bang et al. 1994, Burchardt et al. 1999). We examined this in further detail via our cell models which recapitulate the cellular phenotype of ENZR and neuroendocrine PCa. Our modeling of ENZR provides a unique research platform as $42 \mathrm{D}^{\text {ENZR }}$ and $42 \mathrm{FENZR}^{\mathrm{EN}}$ cells proliferate under the pressure of ENZ, remain AR positive but PSA negative and also demonstrate markers of terminal NE differentiation (Bishop et al. 2017). We show that maintaining NE marker status in these cell lines is dependent on PEG10 expression, meaning knockdown of PEG10 resulted in the attenuation of NE marker status while PEG10 overexpression alone was not sufficient to induce NE differentiation in LNCaP cells. These findings suggest that PEG10 is not a driver of NEPC but is crucial in maintaining this phenotype. Moreover, PEG10 inhibition reduced proliferation in vitro and attenuates tumor growth in vivo making it an attractive therapeutic target in advanced prostate cancers where NE differentiation or growth may be PEG10 dependent. Our results were supported by a recent study that shows inhibition of PEG10 may be a novel treatment approach in a subset of bladder cancer (Kawai et al. 2017).

In summary, this study adds to our understanding of the mechanisms involved in tNEPC and the molecular underpinnings driving neuroendocrine differentiation. We highlight the non-canonical role that AR plays in mediating neuroendocrine transdifferentiation, namely its effect to repress the NE phenotype in prostate cancer by suppressing PEG10 expression. Utilizing in vitro, in vivo study design and corroborating our findings with human cancer databases, we have shown that PEG1O is one of the few genes that have been identified in multiple datasets as being upregulated during and following transdifferentiation (Beltran et al. 2011, 2016, Akamatsu et al. 2015) and a potential therapeutic target in NEPC.

\section{Supplementary data}

This is linked to the online version of the paper at https://doi.org/10.1530/ JME-18-0226.

\section{Declaration of interest}

The authors declare that there is no conflict of interest that could be perceived as prejudicing the impartiality of the research reported.

\section{Funding}

This research was funded by Prostate Cancer Canada Team Grant (2013).

\section{Author contribution statement}

$\mathrm{S} \mathrm{K}$ :Experimental design, data acquisition, statistical analysis; D T and S V: manuscript preparation, data acquisition, data analysis; $\mathrm{S} B$ : manuscript preparation; S A, P T and C C: manuscript editing; J L B: experimental design, data analysis, manuscript preparation; A Z: experimental design, study overview, data analysis, manuscript preparation.

\section{References}

Aggarwal R, Huang J, Alumkal JJ, Zhang L, Feng FY, Thomas GV, Weinstein AS, Friedl V, Zhang C, Witte ON, et al. 2018 Clinical and genomic characterization of treatment-emergent small-cell neuroendocrine prostate cancer: a multi-institutional prospective study. Journal of Clinical Oncology 36 2492-2503. (https://doi. org/10.1200/JCO.2017.77.6880)

Akamatsu S, Wyatt AW, Lin D, Lysakowski S, Zhang F, Kim S, Tse C, Wang K, Mo F, Haegert A, et al. 2015 The placental gene PEG10 promotes progression of neuroendocrine prostate cancer. Cell Reports 12 922-936. (https://doi.org/10.1016/j.celrep.2015.07.012)
(C) 2019 Society for Endocrinology Published by Bioscientifica Ltd. Printed in Great Britain 
Akamatsu S, Inoue T, Ogawa O \& Gleave ME 2018 Clinical and molecular features of treatment-related neuroendocrine prostate cancer. International Journal of Urology 25 345-351. (https://doi. org/10.1111/iju.13526)

Balbas MD, Evans MJ, Hosfield DJ, Wongvipat J, Arora VK, Watson PA, Chen Y, Greene GL, Shen Y \& Sawyers CL 2013 Overcoming mutation-based resistance to antiandrogens with rational drug design. ELife 2 e00499. (https://doi.org/10.7554/eLife.00499)

Bang YJ, Pirnia F, Fang WG, Kang WK, Sartor O, Whitesell L, Ha MJ, Tsokos M, Sheahan MD, Nguyen P, et al. 1994 Terminal neuroendocrine differentiation of human prostate carcinoma cells in response to increased intracellular cyclic AMP. PNAS 91 5330-5334. (https://doi.org/10.1073/pnas.91.12.5330)

Beltran H, Rickman DS, Park K, Chae SS, Sboner A, Macdonald TY, Wang Y, Sheikh KL, Terry S, Tagawa ST, et al. 2011 Molecular characterization of neuroendocrine prostate cancer and identification of new drug targets. Cancer Discovery 1 487-495. (https://doi. org/10.1158/2159-8290.CD-11-0130)

Beltran H, Tomlins S, Aparicio A, Arora V, Rickman D, Ayala G, Huang J, True L, Gleave ME, Soule H, et al. 2014 Aggressive variants of castration-resistant prostate cancer. Clinical Cancer Research 20 2846-2850. (https://doi.org/10.1158/1078-0432.CCR-13-3309)

Beltran H, Prandi D, Mosquera JM, Benelli M, Puca L, Cyrta J, Marotz C, Giannopoulou E, Chakravarthi BV, Varambally S, et al. 2016 Divergent clonal evolution of castration-resistant neuroendocrine prostate cancer. Nature Medicine 22 298-305. (https://doi. org/10.1038/nm.4045)

Beltran H, Wyatt AW, Chedgy EC, Donoghue A, Annala M, Warner EW, Beja K, Sigouros M, Mo F, Fazli L, et al. 2017 Impact of therapy on genomics and transcriptomics in high-risk prostate cancer treated with neoadjuvant docetaxel and androgen deprivation therapy. Clinical Cancer Research 23 6802-6811. (https://doi. org/10.1158/1078-0432.CCR-17-1034)

Bishop JL, Davies A, Ketola K \& Zoubeidi A 2015 Regulation of tumor cell plasticity by the androgen receptor in prostate cancer. EndocrineRelated Cancer 22 R165-R182. (https://doi.org/10.1530/ERC-15-0137)

Bishop JL, Thaper D, Vahid S, Davies A, Ketola K, Kuruma H, Jama R, Nip KM, Angeles A, Johnson F, et al. 2017 The master neural transcription factor BRN2 is an androgen receptor-suppressed driver of neuroendocrine differentiation in prostate cancer. Cancer Discovery 7 54-71. (https://doi.org/10.1158/2159-8290.CD-15-1263)

Burchardt T, Burchardt M, Chen MW, Cao Y, De La Taille A, Shabsigh A, Hayek O, Dorai T \& Buttyan R 1999 Transdifferentiation of prostate cancer cells to a neuroendocrine cell phenotype in vitro and in vivo. Journal of Urology 162 1800-1805. (https://doi.org/10.1016/S00225347(05)68241-9)

CCSsACoC S 2017 Canadian Cancer Statistics 2017. Toronto, ON: Canadian Cancer Society.

Dardenne E, Beltran H, Benelli M, Gayvert K, Berger A, Puca L, Cyrta J, Sboner A, Noorzad Z, Macdonald T, et al. 2016 N-Myc induces an EZH2-mediated transcriptional program driving neuroendocrine prostate cancer. Cancer Cell 30 563-577. (https://doi.org/10.1016/j. ccell.2016.09.005)

Davies AH, Beltran H \& Zoubeidi A 2018 Cellular plasticity and the neuroendocrine phenotype in prostate cancer. Nature Reviews: Urology 15 271-286. (https://doi.org/10.1038/nrurol.2018.22)

Grasso CS, Wu YM, Robinson DR, Cao X, Dhanasekaran SM, Khan AP, Quist MJ, Jing X, Lonigro RJ, Brenner JC et al. 2012 The mutational landscape of lethal castration-resistant prostate cancer. Nature $\mathbf{4 8 7}$ 239-243. (https://doi.org/10.1038/nature11125)

Joseph JD, Lu N, Qian J, Sensintaffar J, Shao G, Brigham D, Moon M, Maneval EC, Chen I, Darimont B, et al. 2013 A clinically relevant androgen receptor mutation confers resistance to second-generation antiandrogens enzalutamide and ARN-509. Cancer Discovery 3 1020-1029. (https://doi.org/10.1158/2159-8290.CD-13-0226)
Kawai Y, Zhang F, Akamatsu S, Hayashi T, Imada K, Beraldi E, Seiler R, Leong J, Oo H, Moskalev I, et al. 2017 MP65-03 inhibition of Peg10 may be a novel treatment strategy for a subset of bladder cancer. Journal of Urology 197 E853-E853. (https://doi.org/10.1016/j. juro.2017.02.1997)

Korpal M, Korn JM, Gao X, Rakiec DP, Ruddy DA, Doshi S, Yuan J, Kovats SG, Kim S, Cooke VG, et al. 2013 An F876L mutation in androgen receptor confers genetic and phenotypic resistance to MDV3100 (enzalutamide). Cancer Discovery 3 1030-1043. (https:// doi.org/10.1158/2159-8290.CD-13-0142)

Kuruma H, Matsumoto H, Shiota M, Bishop J, Lamoureux F, Thomas C, Briere D, Los G, Gleave M, Fanjul A, et al. 2013 A novel antiandrogen, compound 30, suppresses castration-resistant and MDV3100-resistant prostate cancer growth in vitro and in vivo. Molecular Cancer Therapeutics 12 567-576. (https://doi. org/10.1158/1535-7163.MCT-12-0798)

Li Y, Chan SC, Brand LJ, Hwang TH, Silverstein KA \& Dehm SM 2013 Androgen receptor splice variants mediate enzalutamide resistance in castration-resistant prostate cancer cell lines. Cancer Research $\mathbf{7 3}$ 483-489. (https://doi.org/10.1158/0008-5472.CAN-12-3630)

Li X, Xiao R, Tembo K, Hao L, Xiong M, Pan S, Yang X, Yuan W, Xiong J \& Zhang Q 2016 PEG10 promotes human breast cancer cell proliferation, migration and invasion. International Journal of Oncology 48 1933-1942. (https://doi.org/10.3892/ ijo.2016.3406)

Ono R, Nakamura K, Inoue K, Naruse M, Usami T, Wakisaka-Saito N, Hino T, Suzuki-Migishima R, Ogonuki N, Miki H, et al. 2006 Deletion of Peg10, an imprinted gene acquired from a retrotransposon, causes early embryonic lethality. Nature Genetics $\mathbf{3 8}$ 101-106. (https://doi.org/10.1038/ng1699)

Peng YP, Zhu Y, Yin LD, Zhang JJ, Wei JS, Liu X, Liu XC, Gao WT, Jiang KR \& Miao Y 2017 PEG10 overexpression induced by E2F-1 promotes cell proliferation, migration, and invasion in pancreatic cancer. Journal of Experimental and Clinical Cancer Research 3630. (https://doi.org/10.1186/s13046-017-0500-x)

Rajan P, Sudbery IM, Villasevil ME, Mui E, Fleming J, Davis M, Ahmad I, Edwards J, Sansom OJ, Sims D, et al. 2014 Next-generation sequencing of advanced prostate cancer treated with androgendeprivation therapy. European Urology 66 32-39. (https://doi. org/10.1016/j.eururo.2013.08.011)

Ribarska T, Bastian KM, Koch A \& Schulz WA 2012 Specific changes in the expression of imprinted genes in prostate cancer - implications for cancer progression and epigenetic regulation. Asian Journal of Andrology 14 436-450. (https://doi.org/10.1038/aja.2011.160)

Robertson AG, Kim J, Al-Ahmadie H, Bellmunt J, Guo GW, Cherniack AD, Hinoue T, Laird PW, Hoadley KA, Akbani R, et al. 2017 Comprehensive molecular characterization of muscle-invasive bladder cancer. Cellule 171 540.e25-556.e25. (https://doi. org/10.1016/j.cell.2017.09.007)

Rousseaux S, Debernardi A, Jacquiau B, Vitte AL, Vesin A, NagyMignotte H, Moro-Sibilot D, Brichon PY, Lantuejoul S, Hainaut P, et al. 2013 Ectopic activation of germline and placental genes identifies aggressive metastasis-prone lung cancers. Science Translational Medicine 5 186ra66. (https://doi.org/10.1126/ scitranslmed.3005723)

Scher HI, Fizazi K, Saad F, Taplin ME, Sternberg CN, Miller K, De Wit R, Mulders P, Chi KN, Shore ND, et al. 2012 Increased survival with enzalutamide in prostate cancer after chemotherapy. New England Journal of Medicine 367 1187-1197. (https://doi.org/10.1056/ NEJMoa1207506)

Tan HL, Sood A, Rahimi HA, Wang W, Gupta N, Hicks J, Mosier S, Gocke CD, Epstein JI, Netto GJ, et al. 2014 Rb loss is characteristic of prostatic small cell neuroendocrine carcinoma. Clinical Cancer Research 20 890-903. (https://doi.org/10.1158/1078-0432. CCR-13-1982) 
Terry S \& Beltran H 2014 The many faces of neuroendocrine differentiation in prostate cancer progression. Frontiers in Oncology 4 60. (https://doi.org/10.3389/fonc.2014.00060)

Tzelepi V, Zhang J, Lu JF, Kleb B, Wu G, Wan X, Hoang A, Efstathiou E, Sircar K, Navone NM, et al. 2012 Modeling a lethal prostate cancer variant with small-cell carcinoma features. Clinical Cancer Research 18 666-677. (https://doi.org/10.1158/1078-0432.CCR-11-1867)

Wright ME, Tsai MJ \& Aebersold R 2003 Androgen receptor represses the neuroendocrine transdifferentiation process in prostate cancer cells.
Molecular Endocrinology 17 1726-1737. (https://doi.org/10.1210/ me.2003-0031)

Zhang X, Coleman IM, Brown LG, True LD, Kollath L, Lucas JM, Lam HM, Dumpit R, Corey E, Chery L, et al. 2015 SRRM4 expression and the loss of REST activity may promote the emergence of the neuroendocrine phenotype in castration-resistant prostate cancer. Clinical Cancer Research 21 4698-4708. (https://doi.org/10.1158/1078-0432. CCR-15-0157)

Received in final form 13 March 2019

Accepted 23 April 2019

Accepted Preprint published online 23 April 2019
(C) 2019 Society for Endocrinology Published by Bioscientifica Ltd. Printed in Great Britain 